from controls in schizophrenic-like or dissociative symptoms.

Discussion: The dose-dependent hypopriming effect at the long SOA induced by acute ketamine was indicative of controlled processing impairments. In ketamine users, there was also an indication of controlled processing impairments and a suggestion that longterm ketamine abuse results in damage to the semantic store.

\section{Behavioural and fMRI evidence of semantic category deficits in schizophrenia}

\section{S Rossell, I Labuschagne}

Mental Health Research Institute of Victoria, Melbourne, Australia

Background: Abnormalities in semantic processing are commonly proposed to be central to cognitive abnormalities and thought disturbances in schizophrenia. Deficits have been reported on a range of tasks including a categorization task. The current study investigated the underlying neural substrates involved during categorization.

Method: A revised version of Chen et al.'s (1994) categorization task was used. The task consisted of 18 categories with five different exemplar words (ie high frequency, low frequency, borderline, related but outside category and unrelated) selected for each category. Subjects were asked to say whether exemplars were or were not part of the category. Data for each exemplar type were examined; this included behavioural accuracy and an event-related analysis of the functional magnetic resonance imaging data using SPM2.

Results: Behaviourally, patients with schizophrenia had difficulty categorizing related words, while the controls had most difficulty with borderline examples. Performance in the controls was related to activity in the left inferior frontal, left inferior occipital/posterior temporal, bilateral precunues and the cerebellum; areas typically reported during semantic processing. Even when behavioural performance on some of the category types was no different to control performance, the patients with schizophrenia did not show any activation of this network.

Conclusions: The imaging data showed impairments in the distributed frontal temporal network that is engaged in the representation and processing of meaning of words, text and discourse. It is these abnormalities that may underlie schizophrenic thought disturbance.

\section{Jumping to conclusions in delusions: fact or fallacy?}

\author{
S Rossell, A O'Regan, N Joshua
}

Mental Health Research Institute, Melbourne, Australia

Background: A jumping-to-conclusions (JTC) bias refers to the gathering of minimal data when making probabilistic judgments and has been associated with delusion formation. Approximately $50 \%$ of patients who experience delusions have previously been shown to have a JTC bias. However, the literature is fraught with methodological differences. This study sought to address one of these differences by examining state and trait delusions using large groups of patients with psychosis.

Methods: Three matched groups (patients with bipolar disorder, patients with schizophrenia and healthy controls) completed the standard beads probabilistic reasoning task and two emotional variants, which examined reasoning using personality and health traits. For each of the three tasks, two probability ratios were used, 85:15 and 60:40. JTC was defined, using the Institute of Psychiatry, London, criteria, as requiring two or less draws. Patients were divided into those with state and trait delusions.

Results: On the beads $85: 15$ task, only $4 \%$ of patients with schizophrenia and $0 \%$ of patients with bipolar disorder showed a JTC bias. The emotional variants produced a similar degree of JTC bias. The 60:40 ratio resulted in an even smaller proportion of JTC. There were no state-trait differences.

Discussion: Two groups of Australian patients with psychosis (schizophrenia and bipolar disorder) did not show a JTC bias. The authors discuss possible explanations for the discrepancy of these findings with the literature. These include the following: are Australians more indecisive or cautious, how state and trait delusions influence performance, and methodological problems with the task itself.

\section{Building and evaluating effective mental health networks in rural communities}

\author{
G Sartore', B Kelly', L Fragar², J Fuller³ , H Stain'1, \\ A Tonna', G Pollard' \\ 'Centre for Rural and Remote Mental Health, University of Newcastle; \\ ${ }^{2}$ Australian Centre for Agricultural Health and Safety, The University of Sydney; \\ and ${ }^{3}$ Northern Rivers University Department of Rural Health, \\ The University of Sydney, Sydney, Australia
}

Background: Rural communities suffer significant disadvantage in accessing mental health services. Conversely, these communities may have greater capacity 